\title{
Corrigendum to: Positivity theorems for solid-angle polynomials
}

\author{
Matthias Beck' ${ }^{1}$. Sinai Robins ${ }^{2}$ - Steven V. Sam ${ }^{3}$
}

Received: 27 June 2015 / Accepted: 28 June 2015 / Published online: 24 July 2015

(C) The Managing Editors 2015

The proofs of [BRS, Theorems 3,4] given in [BRS, §6] contain an error, and indeed, the statements are not correct in the level of generality given there. We thank Katharina Jochemko and Raman Sanyal for pointing out the mistake to us. To be precise, the mistake lies in the assertion that equation (9) implies that

$$
\sigma_{\operatorname{cone}(\mathcal{P})}(\mathbf{z})=\sum_{j=1}^{m} \sigma_{\mathbf{v}+\mathcal{K}_{j}}(\mathbf{z}) .
$$

Unraveling this, the series of implications one gets is

$$
\begin{aligned}
\sum_{j=1}^{m} \sigma_{\mathbf{v}+\mathcal{K}_{j}}(\mathbf{z}) & =\sum_{j=1}^{m} \sum_{\mathbf{m} \in\left(\mathbf{v}+\mathcal{K}_{j}\right) \cap \mathbf{Z}^{d+1}} v\left(\mathbf{v}+\mathcal{K}_{j}, \mathbf{m}\right) \mathbf{z}^{\mathbf{m}} \\
& =\sum_{\mathbf{m} \in(\mathbf{v}+\operatorname{cone}(\mathcal{P})) \cap \mathbf{Z}^{d+1}} v(\mathbf{v}+\operatorname{cone}(\mathcal{P}), \mathbf{m}) \mathbf{z}^{\mathbf{m}}
\end{aligned}
$$

We had implicitly replaced $v(\mathbf{v}+\operatorname{cone}(\mathcal{P}), \mathbf{m})$ with $v(\mathbf{v}+\operatorname{cone}(\mathcal{P}), \mathbf{v}+\mathbf{m})$ and used translation-invariance to replace the second quantity with $v(\operatorname{cone}(\mathcal{P}), \mathbf{m})$. However, $v(\mathbf{v}+\operatorname{cone}(\mathcal{P}), \mathbf{m})$ and $v(\mathbf{v}+\operatorname{cone}(\mathcal{P}), \mathbf{v}+\mathbf{m})$ are in general distinct without further properties on $v$.

Steven V. Sam

svs@math.berkeley.edu

1 Department of Mathematics, San Francisco State University, San Francisco, CA 94132, USA

2 Division of Mathematical Sciences, School of Physical and Mathematical Sciences, Nanyang Technological University, Singapore 637371, Singapore

3 Department of Mathematics, University of California, Berkeley, CA 94720, USA 
We refer to [JS] for a corrected version of those theorems as well as counterexamples.

Finally, the valuation $v^{\prime}(K, \mathbf{x})=\#\left(K \cap\{\mathbf{x}\} \cap \mathbf{Z}^{d}\right)$ is not translation-invariant, as was stated in the introduction of [BRS].

\section{References}

BRS. Beck, M., Robins, S., Sam, S.V.: Positivity theorems for solid-angle polynomials. Beitr. Algebra Geom. 51(2), 493-507 (2010). arXiv:0906.4031v2

JS. Jochemko, K., Sanyal, R.: Combinatorial positivity of translation-invariant valuations (2015). arXiv: $1505.07440 \mathrm{v} 1$ 